1. Moore MJ, Proudfoot NJ. Pre-mRNA processing reaches back to transcription and ahead to translation. Cell. 2009;136(4):688-700

2. Wang ET, et al. Alternative isoform regulation in human tissue transcriptomes. Nature. 2008; 456(7221):470-476.

3. Pan Q, Shai O, Lee LJ, Frey BJ, Blencowe BJ. Deep surveying of alternative splicing complexity in the human transcriptome by high-throughput sequencing. Nat Genet. 2008;40(12):1413-1415.

4. Han SP, Tang YH, Smith R. Functional diversity of the hnRNPs: past, present and perspectives. Biochem J. 2010;430(3):379-392

5. Black DL. Mechanisms of alternative pre-messenger RNA splicing. Annu Rev Biochem. 2003; 72:291-336

6. Faustino NA, Cooper TA. Pre-mRNA splicing and human disease. Genes Dev. 2003;17(4):419-437.

7. Goehe RW, et al. hnRNP L regulates the tumorigenic capacity of lung cancer xenografts in mice via caspase-9 pre-mRNA processing. J Clin Invest. 2010;120(11):3923-3939.

8. Seol DW, Billiar TR. A caspase-9 variant missing the catalytic site is an endogenous inhibitor of apoptosis. J Biol Chem. 1999;274(4):2072-2076.

9. Philchenkov A, Zavelevich M, Kroczak TJ, Los M Caspases and cancer: mechanisms of inactivation and new treatment modalities. Exp Oncol. 2004;26(2):82-97.

10. Pio R, Montuenga LM. Alternative splicing in lung cancer. JThorac Oncol. 2009;4(6):674-678.

11. Skotheim RI, Nees M. Alternative splicing in cancer: noise, functional, or systematic? Int J Biochem Cell Biol. 2007;39(7-8):1432-1449.

12. Boukakis G, Patrinou-Georgoula M, Lekarakou M, Valavanis C, Guialis A. Deregulated expression of hnRNP A/B proteins in human non-small cell lung cancer: parallel assessment of protein and mRNA levels in paired tumor/non-tumor tissues. $B M C$ Cancer. 2010;10:434.

13. Clower CV, Chatterjee D, Wang Z, Cantley LC, Vander Heiden MG, Krainer AR. The alternative splicing repressors hnRNP A1/A2 and PTB influence pyruvate kinase isoform expression and cell metabolism. Proc Natl Acad Sci U S A. 2010; 107(5):1894-1899.

14. David CJ, Chen M, Assanah M, Canoll P, Manley JL. HnRNP proteins controlled by c-Myc deregulate pyruvate kinase mRNA splicing in cancer. Nature. 2010;463(7279):364-368

15. Hung LH, Heiner M, Hui J, Schreiner S, Benes V, Bindereif A. Diverse roles of hnRNP L in mammalian mRNA processing: a combined microarray and RNAi analysis. RNA. 2008;14(2):284-296.

16. Hui J, Reither G, Bindereif A. Novel functional role of CA repeats and hnRNP L in RNA stability. RNA. 2003;9(8):931-936.

17. Hui J, Stangl K, Lane WS, Bindereif A. HnRNP L stimulates splicing of the eNOS gene by binding to variable-length CA repeats. Nat Struct Biol. 2003;10(1):33-37.

18. Xiao SH, Manley JL. Phosphorylation of the ASF/ SF2 RS domain affects both protein-protein and protein-RNA interactions and is necessary for splicing. Genes Dev. 1997;11(3):334-344.

19. Shih SC, Claffey KP. Regulation of human vascular endothelial growth factor mRNA stability in hypoxia by heterogeneous nuclear ribonucleoprotein L.J Biol Chem. 1999;274(3):1359-1365.

20. Lynch KW. Regulation of alternative splicing by signal transduction pathways. Adv Exp Med Biol. 2007;623:161-174.

\title{
Pregnancy immunogenetics: NK cell education in the womb?
}

\author{
Peter Parham and Lisbeth A. Guethlein
}

Department of Structural Biology and Department of Microbiology and Immunology, Stanford University, Stanford, California, USA.

\begin{abstract}
During embryo implantation and initiation of pregnancy, uterine NK (uNK) cells engage invasive fetal trophoblasts to remodel vessels that conduct blood to the placenta. This partnership, involving uNK cell receptors that recognize HLA-C ligands on trophoblasts, varies the course of human pregnancy because the genes for both receptors and ligands are extraordinarily diverse. Several pregnancy disorders are attributed to insufficient trophoblast invasion and the limitation it imposes on human reproduction. Previously, a particular combination of fetal HLA-C and maternal inhibitory uNK cell receptor was associated with predisposition for preeclampsia. In this issue of the JCI, Hiby and colleagues extend this correlation to recurrent miscarriage and fetal growth restriction, revealing the common mechanism underlying these common pregnancy syndromes. Equally important, they show that mothers with an activating receptor of similar specificity to the inhibitory receptor are less likely to suffer disordered pregnancy.
\end{abstract}

Although glittering prizes for fundamental immunology emerged from the study of lymphocyte responses to genetic polymorphisms within species (1-5), pregnancy is the only situation in which lymphocytes naturally confront another person's tissue. On embryo implantation and placentation (6), uterine NK (uNK) cells of the mother cooperate with extravillous trophoblasts (EVTs), fetal cells that invade the uterus,

Conflict of interest: The authors have declared that no conflict of interest exists.

Citation for this article: J Clin Invest. doi:10.1172/ JCI44559. where they remove and replace the smooth muscle of maternal spiral arteries. This process converts these small, coiled vessels into voluminous channels that will nourish the growing child. A fine line exists between excessive invasion that damages the mother and inadequate invasion that starves the child. To achieve this balance, interactions must occur between a variety of activating and inhibitory NK cell receptors and their ligands on EVTs. Common disorders of pregnancy - recurrent miscarriage, preeclampsia, and fetal growth restriction - are attributed to inadequate trophoblast invasion (7). Pointing toward the importance of interaction between EVTs and uNK cells, Hiby et al. correlated combinations of fetal HLA and maternal uNK cell receptors with risk of preeclampsia (8). Inevitably, their results, with provocative implications for female mate choice and human reproduction (9), elicited a range of reactions in the biomedical community, from enthusiasm to diffidence to skepticism. In this issue of the JCI, Hiby et al. answer their critics by advancing the immunogenetic analysis to a higher level, extending the epidemiology to embrace other pregnancy disorders, and vividly visualizing the interaction of EVT with uNK cells (10).

\section{Killer immunoglobulin-like receptors of NK cells that recognize HLA-C}

Numerous human diseases, including ankylosing spondylitis (11), a back-bending type of arthritis, and life-threatening hypersensitivities to drugs prescribed as treatment for epilepsy and HIV/AIDS (12), are correlated with the highly polymorphic HLA-A, $-B$, and -C genes of the human major histocompatibility complex. Of their protein products, only HLA-C is expressed by EVT and binds to uNK cell receptors. Although hundreds of HLA-C variants are distinguished worldwide, in the NK 

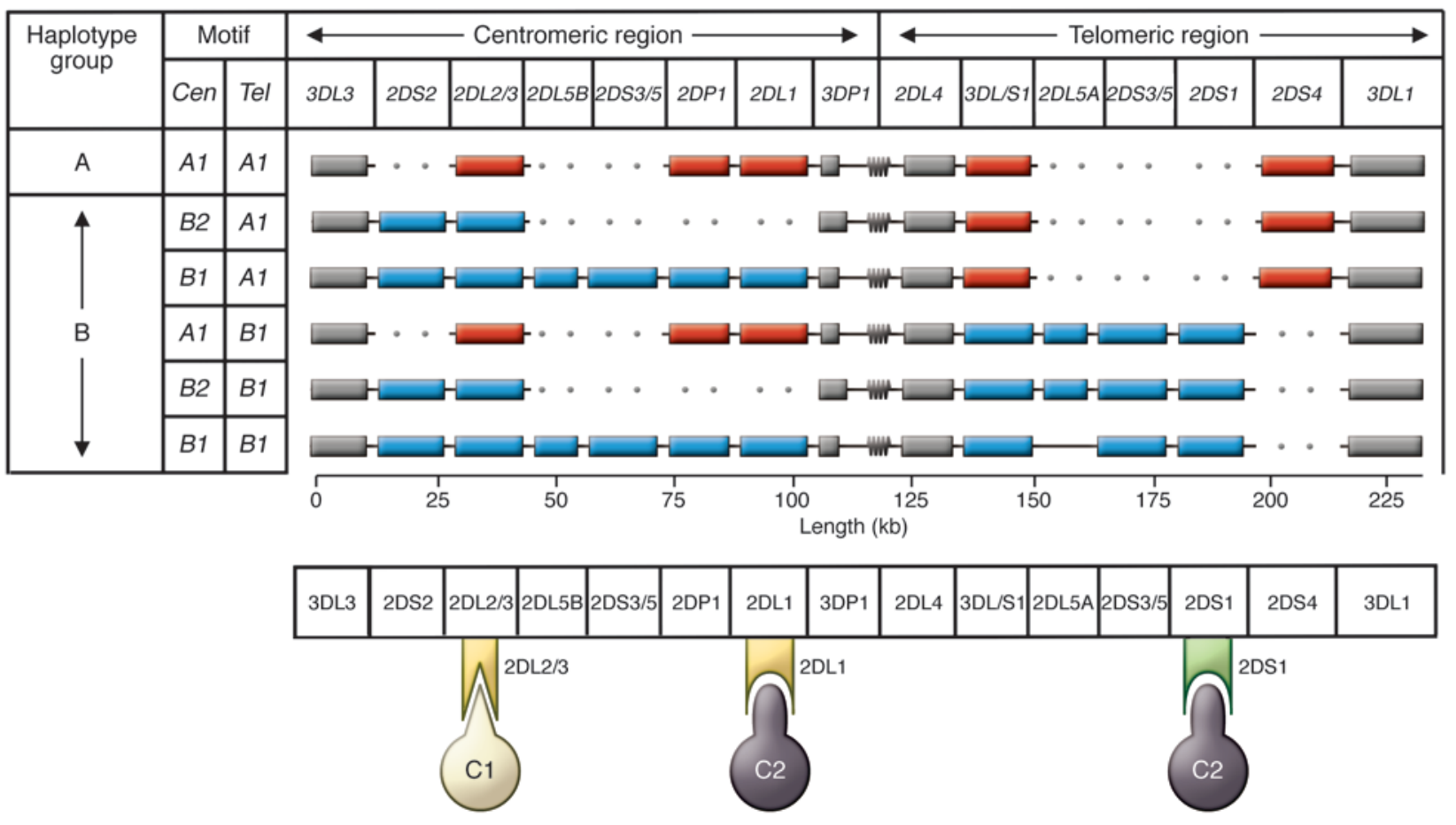

Figure 1

The human KIR locus consists of centromeric and telomeric regions that recombine to produce $A$ and $B$ haplotypes with a distinctive gene content. Structures of common haplotypes are shown in the top panel. Gray boxes indicate conserved framework genes; red boxes indicate genes characteristic of $A$ haplotypes; blue boxes indicate $B$ haplotype-specific genes. The centromeric (Cen) and telomeric (Tel) gene-content motifs are listed at left. The symbol at the boundary between the centromeric and telomeric regions represents a repetitive sequence, the site for meiotic recombination that has shuffled the centromeric and telomeric motifs. The bottom panel illustrates HLA-C-specific KIRs and their specificities for the $\mathrm{C} 1$ and $\mathrm{C} 2$ epitopes. Yellow indicates inhibitory receptors KIR2DL2/3 and KIR2DL1; green indicates the activating receptor KIR2DS1.

cell world they are routinely boiled down to two functional groups, defined by the presence of asparagine or lysine at position 80 in the protein sequence (13). The former determines the $\mathrm{C} 1$ epitope (also called HLA-C1), the latter the C2 epitope (HLA-C2). These epitopes are ligands for different members of the family of variable NK cell receptors known as killer immunoglobulin-like receptors (KIRs) (Figure 1 and ref. 14). Each KIR is either an activating or an inhibitory receptor that works as part of a coordinated team to diversify the functions of NK cells. Recognizing C2 are inhibitory KIR2DL1 and activating KIR2DS1. Although C1 is recognized by inhibitory KIR2DL2 and KIR2DL3, there is no corresponding activating receptor. It is puzzling that KIR2DS2 was once that activating receptor, but at some ancient time in human history it became subject to natural selection that reduced $\mathrm{C} 1$ avidity until it was no longer detectable (15). Like HLA, the KIR gene family on human chromosome 19 exhibits extraordinary population diversity, combining haplotypic differences in KIR gene content with allelic polymor- phism. As achieved for HLA-C, functional KIR diversity is reducible to two functionally distinctive haplotype groups, KIR $A$ and $B$, distinguished by the presence and absence of particular subsets of KIR genes (Figure 1 and ref. 14).

\section{Fetal HLA-C2 combined with maternal KIR AA predispose to pregnancy disorders}

Six years ago, Hiby et al. reported that mothers homozygous for KIR A haplotypes (genotype $A A$ ) were at greater risk for preeclampsia than mothers having one $(A B)$ or two $(B B)$ KIR $B$ haplotypes (8). The risk further increased for the subset of KIR $A A$ mothers carrying babies bearing the $\mathrm{C} 2$ epitope. The presence of the gene encoding the inhibitory $\mathrm{C} 2$ receptor (KIR2DL1) and absence of the gene encoding the activating C2 receptor (KIR2DS1) are characteristic of $A$ haplotypes (Figure 1). This epidemiological correlation pointed to the interaction between C2 on EVTs and KIR2DL1 on uNK cells as a factor contributing to preeclampsia. In this issue of the JCI, Hiby et al. demonstrate that the same genetic combination of fetal $\mathrm{C} 2$ and maternal KIR $A A$ also predisposes to recurrent miscarriage and fetal growth restriction (10). Not only do these results support a common mechanism that underpins the three most common pregnancy disorders, but they also allowed the authors to combine their three disease cohorts, permitting more powerful genetic analysis for investigating the protection afforded by maternal KIR B haplotype genes.

\section{Activating C2 receptor KIR2DS1 protects against pregnancy disorder}

Whereas KIR A haplotypes have fixed gene content, $B$ haplotypes are variable. Characteristic $B$ haplotype genes can be present in the centromeric region of the KIR locus, the telomeric region, or both (Figure 1). By comparing the pregnancies of mothers whose $B$ haplotype genes are only centromerically or telomerically located, Hiby et al. found that the bulk of protection tracked with the telomeric genes: KIR3DS1, KIR2DL5, KIR2DS3/5, and KIR2DS1 (10). Although genetic analysis cannot distinguish between them, because of very strong 
linkage disequilibrium, functional studies argue for KIR2DS1 as the protective gene. KIR2DS1 is a well-defined C2 receptor, while KIR3DS1, KIR2DL5, and KIR2DS3/5 remain enigmatic receptors of baffling ligand specificity. Furthermore, protection increases when the fetus expresses the C2 ligand for KIR2DS1. Having previously established that disordered pregnancy is more likely when the fetus expresses C2 and the mother has only inhibitory C2 receptors, Hiby et al. now demonstrate in complementary fashion that maternal activating $\mathrm{C} 2$ receptors are protective (10). How this plays out at the cellular level remains unknown, but a simple working model is that dominance of the inhibitory C2 receptor reduces NK cell stimulation of EVTs and the depth to which they invade the uterus, whereas presence of an activating C2 receptor serves as a counterbalance to increase EVT stimulation and the depth of uterine invasion. A precedent for such a mechanism has been established for many other pairs of activating and inhibitory immune system receptors (16).

\section{Paternal inheritance of HLA-C2 carries greater risk than maternal inheritance}

The KIR locus is turned on late in NK cell development, in a stochastic fashion that produces a diversity of NK cells expressing different combinations of KIRs. Developmental interactions of the KIRs with HLA class I determine the strength and specificity with which mature NK cells respond to the perturbations from self HLA class I present on infected, malignant, or HLAdisparate fetal cells. This instruction of NK cells by HLA class I is called education. It is pertinent here that $\mathrm{C} 2$ recognition by KIR2DL1 (17) and KIR2DS1 (18) educate immature NK cells to respond to cells exhibiting unusual expression of the C2 epitope. While most people have KIR2DL1, only a minority have C2. Regardless of the presence or absence of C2, KIR2DL1 is expressed by some mature NK cells. But in the absence of C2, KIR2DL1 cannot participate in education and appears to be nonfunctional. In this context it was important for Hiby et al. to compare the influence on pregnancy of maternally and paternally inherited fetal C2. With maternal inheritance, the mother's uNK cells were exposed to C2 during their development and prior to pregnancy, but this was not so for paternal inheritance (10). After demonstrating that EVTs express both the maternally and paternally inherited $H L A-C$ alleles, the authors analyzed the subset of pregnancies in which the fetus carried one copy of C2, comparing the effect of maternal or paternal origin. Disease was more prevalent when the fetus expressed paternal C2 (10). A less incisive approach, but one empowered by the full disease cohort, showed greater disease risk when the fetus had more copies of $\mathrm{C} 2$ than did the mother. Both analyses point to the dominant effect of $\mathrm{C} 2$ inherited from the father, indicating that the uNK cell population educated by a woman's own C2 is compatible with that same $\mathrm{C} 2$ when subsequently expressed by EVTs during pregnancy.

\section{Do mother's NK cells become educated during pregnancy to paternally inherited HLA-C?}

Preeclampsia has famously been described as a problem of first pregnancies, but not subsequent ones (19). Although controversial, such observations raise the intriguing possibility that KIR AA mothers adapt to the immunogenetic disadvantage of a fetus expressing C2. During a first pregnancy, the nine-month exposure to C2 could permanently modify the developmental program that educates uNK cells and determines their receptor repertoire. Alternatively, passage of C2-expressing fetal cells into the maternal circulation could establish microchimerism that actively educates maternal NK cells to work with paternal C2. The similarly controversial suggestion that maternal adaptation following a first pregnancy may only pertain to subsequent pregnancies with the same father (19) implies that the C2 epitopes carried by the numerous HLA-C variants with lysine 80 are not all equivalent, as is evident from their wide-ranging avidities for KIR2DS1 and KIR2DL1 (15).

\section{Worldwide impact of natural selection by pregnancy disorders}

HLA class I and KIR are diverse gene families that interact functionally but segregate independently in human populations. Thus many individuals express KIR, for which they lack HLA class I ligands, and vice versa. Nonetheless, at the level of the population there is compelling evidence for coevolution of interacting pairs of KIR and HLA class I $(20,21)$. As Hiby et al. originally proposed (8), there is worldwide a striking inverse correlation between the frequencies of $\mathrm{C} 2$ and the KIR $A$ haplotype. This correlation ranges from populations with a high frequency of $A$ haplotypes and a low frequency of the C2 epitope to those who have a high frequency of $B$ haplotypes and a high frequency of C2. This heavy footprint, which acts universally to reduce the frequency of KIR AA mothers carrying C2 babies, is the smoking gun that indicates common pregnancy disorders as a major selective force throughout human history, as they remain today in the developing world (22). In the absence of modern medicine, recurrent miscarriage prevents reproduction, fetal growth restriction produces less competitive offspring, and preeclampsia, with its endpoint of eclampsia, kills both mother and child. Equally impressive, such selection has neither eliminated HLA-C2 nor KIR A in any surviving human population (21). This reflects balancing selection on both the $\mathrm{C} 1 / \mathrm{C} 2$ and $\mathrm{KIR} A B$ dimorphisms, each alternative having significant costs and benefits. The strength and persistence of the balancing selection reflects the fact that NK cells have two seemingly different functions, with their own distinctive needs and competing demands. Besides their role in the wombs of women during reproduction, NK cells are major players for both men and women in the systemic, innate immune response to infection. But that is another story.

\section{Acknowledgments}

Research in the authors' laboratory was supported by NIH grants AI22039 and AI17892 to P. Parham.

Address correspondence to: Peter Parham, Department of Structural Biology, Fairchild D-157, 299 Campus Dr. West, Stanford, California 94305, USA. Phone: 650.723.7456; Fax: 650.723.8464; E-mail: peropa@stanford.edu.

1. Billingham RE, Brent L, Medawar PB. Actively acquired tolerance of foreign cells. Nature. 1953;172(4379):603-606.

2. Snell GD. Studies in histocompatibility. Science. 1981;213(4504):172-178.

3. Zinkernagel RM, Doherty PC. The discovery of MHC restriction. Immunol Today. 1997;18(1):14-17.

4. Thomas ED, Blume KG. Historical markers in the development of allogeneic hematopoietic cell transplantation. Biol Blood Marrow Transplant. 1999;5(6):341-346.

5. Karre K. Natural killer cell recognition of missing self. Nat Immunol. 2008;9(5):477-480.

6. Moffett A, Loke C. Immunology of placentation in eutherian mammals. Nat Rev Immunol. 2006; 6(8):584-594.

7. Pijnenborg R, Brosens I, Romero R, eds. Placental Bed Disorders. Cambridge, United Kingdom: Cambridge University Press; 2010.

8. Hiby SE, et al. Combinations of maternal KIR and fetal HLA-C genes influence the risk of preeclampsia and reproductive success. J Exp Med. 2004; 
200(8):957-965.

9. Saito S, Takeda Y, Sakai M, Nakabayahi M, Hayakawa $\mathrm{S}$. The incidence of pre-eclampsia among couples consisting of Japanese women and Caucasian men. J Reprod Immunol. 2006;70(1-2):93-98.

10. Hiby SE, et al. Maternal activating KIRs protect against human reproductive failure mediated by fetal HLA-C2. J Clin Invest. 2010;120(11):4102-4110.

11. Tam LS, Gu J, Yu D. Pathogenesis of ankylosing spondylitis. Nat Rev Rheumatol. 2010;6(7):399-405.

12. Chung WH, Hung SI, Chen YT. Human leukocyte antigens and drug hypersensitivity. Curr Opin Allergy Clin Immunol. 2007;7(4):317-323.

13. Mandelboim O, et al. Protection from lysis by natural killer cells of group 1 and 2 specificity is mediated by residue 80 in human histocompatibility leu- kocyte antigen $\mathrm{C}$ alleles and also occurs with empty major histocompatibility complex molecules. J Exp Med. 1996;184(3):913-922.

14. Parham P. MHC class I molecules and KIRs in human history, health and survival. Nat Rev Immunol. 2005;5(3):201-214

15. Moesta AK, GraefT, Abi-Rached L, Older Aguilar AM, Guethein LA, Parham P. Humans differ from other hominids in lacking an activating NK cell receptor that recognizes the $\mathrm{C} 1$ epitope of MHC class I. J Immunol. 2010;185(7):4233-4237.

16. Barclay AN, Hatherley D. The counterbalance theory for evolution and function of paired receptors. Immunity. 2008;29(5):675-678.

17. Anfossi N, et al. Human NK cell education by inhibitory receptors for MHC class I. Immunity. 2006;
25(2):331-342

18. Fauriat C, Ivarsson MA, Ljunggren HG, Malmberg $\mathrm{KJ}$, Michaelsson J. Education of human natural killer cells by activating killer cell immunoglobulin-like receptors. Blood. 2009;115(6):1166-1174.

19. Sibai B, Dekker G, Kupferminc M. Pre-eclampsia. Lancet. 2005;365(9461):785-799.

20. Single RM, et al. Global diversity and evidence for coevolution of KIR and HLA. Nat Genet. 2007; 39(9):1114-1119.

21. Gendzekhadze K, et al. Co-evolution of KIR2DL3 with HLA-C in a human population retaining minimal essential diversity of KIR and HLA class I ligands. Proc Natl Acad Sci US A. 2009;106(44):18692-18697.

22. Duley L. The global impact of pre-eclampsia and eclampsia. Semin Perinatol. 2009;33(3):130-137.

\title{
WT1: a weak spot in KRAS-induced transformation
}

\author{
Silvia Licciulli and Joseph L. Kissil \\ Molecular and Cellular Oncogenesis Program, The Wistar Institute, Philadelphia, Pennsylvania, USA.
}

\begin{abstract}
Activating mutations in the Ras alleles are found frequently in tumors, making the proteins they encode highly attractive candidate therapeutic targets. However, Ras proteins have proven difficult to target directly. Recent approaches have therefore focused on identifying indirect targets to inhibit Ras-induced oncogenesis. For example, RNAi-based negative selection screens to identify genes that when silenced in concert with activating Ras mutations are incompatible with cellular proliferation, a concept known as synthetic lethality. In this issue of the JCI, Vicent et al. report on the identification of Wilms tumor $1(W t 1)$ as a Kras synthetic-lethal gene in a mouse model of lung adenocarcinoma. Silencing of $W t 1$ in cells expressing an endogenous allele of activated $K$ ras triggers senescence in vitro and has an impact on tumor progression in vivo. These findings are of significant interest given previous studies suggesting that the ability of oncogenic Kras to induce senescence versus proliferation depends on its levels of expression.
\end{abstract}

Activating mutations in the Ras genes (KRAS, $H R A S$, and NRAS) are a common occurrence in a broad spectrum of tumors (1). It is therefore not surprising that extensive efforts have been made to develop therapies to directly target oncogenic Ras. However, the Ras proteins have proven to be formidable foes, and success with direct targeting approaches has been limited so far. One strategy to circumvent this issue has been to search for targets that could serve as a potential Achilles' heel to be exploited to have an impact on Ras indirectly. This approach is based on the idea that expression of oncogenic Ras results in tumor cell alterations so that they become dependent on one or more pathways or particular molecular targets, which then

Conflict of interest: The authors have declared that no conflict of interest exists.

Citation for this article: J Clin Invest. doi:10.1172/ JCI44901. represent ideal hits to strike the tumor cells while sparing normal cells (2). The concept of synthetic lethality perfectly fits this idea. Synthetic lethality between two genes occurs when loss of function of one gene results in cell death only in the presence of genetic alteration of the other, while mutation of either gene alone is compatible with viability. Several genes and pathways have been identified by this approach and, interestingly, many of these are not oncogenic themselves but become essential for cells in the tumorigenic state (see below for discussion of specific examples). It is thus clear why a gene that exhibits a synthetic-lethal interaction with activated Ras would represent a highvalue target for the development of therapeutics. In this issue of the JCI, Vicent et al. describe the identification of Wilms tumor 1 (Wt1) as a novel synthetic-lethal gene in a mouse model of Kras-induced tumorigenesis in the lung (3).

\section{Negative selection screens to identify Ras synthetic-lethal interactors}

In the past, synthetic-lethal interactions have been largely studied in model organisms such as yeast, Drosophila melanogaster, and Caenorhabditis elegans. More recently, thanks to the development of RNAi technology (4-6), it has become feasible to extend the concept of synthetic lethality to mammalian cells to identify genes whose loss of function causes growth arrest or cell death (negative selection). Furthermore, the employment of systematic highthroughput platforms has allowed for screening of significant numbers of targets within a relatively short period of time and in an unbiased manner. In the case of Ras, after knocking down one or more specific targets, it is possible to evaluate the effects on viability through side-by-side comparison of cells with and without oncogenic Ras expression. However, the limitations of these approaches should also be noted. For example, the response to the inhibition of any given target will be highly dependent on multiple factors including cell type and screen conditions. Furthermore, to achieve a strong signal-to-background ratio and to control for off-target effects related to RNAi approaches, the experimental conditions of the screening require careful optimization (7). Nevertheless, the power of such approaches has been demonstrated recently by a number of studies (8-10).

Negative selection screens can be conducted using a well-by-well array or a 